\title{
Workflow-based comparison of two Distributed Computing Infrastructures
}

\author{
Johan Montagnat \\ CNRS, Univ. of Nice \\ I3S laboratory \\ johan@i3s.unice.fr \\ Ketan Maheshwari \\ University of Nice \\ CNRS, I3S laboratory \\ ketan@polytech.unice.fr
}

\author{
Tristan Glatard \\ CNRS, INSERM, Univ. of Lyon \\ CREATIS laboratory \\ glatard@creatis.insa-lyon.fr
Eddy Caron INRIA
ENS Lyon, LIP
Eddy.Caron@ens-lyon.fr

\author{
Damien Reimert \\ INRIA \\ ENS Lyon, LIP \\ damien.reimertvasconcellos@ens- \\ lyon.fr \\ Frédéric Desprez
INRIA
ENS Lyon, LIP
Frederic.Desprez@inria.fr
}

\begin{abstract}
Porting applications to Distributed Computing Infrastructures (DCIs) is eased by the use of workflow abstractions. Yet, estimating the impact of the execution DCI on application performance is difficult due to the heterogeneity of the resources available, middleware and operation models. This paper describes a workflow-based experimental method to acquire objective performance comparison criterions when dealing with completely different DCIs. Experiments were conducted on the European EGI and the French Grid'5000 infrastructures to highlight raw performance variations and identify their causes. The results obtained also show that it is possible to conduct experiments on a production infrastructure with similar reproducibility as on an experimental platform.
\end{abstract}

\section{Categories and Subject Descriptors}

C.4 [Computer Systems Organization]: Performance of Systems

\section{General Terms}

Workflows, Distributed Computing Infrastructures, Performance

\section{PROBLEM STATEMENT AND METHOD}

With the maturing of technologies such as grids, large scale Distributed Computing Infrastructures (DCIs) are being increasingly used for addressing various scientific computing needs. Such DCIs deliver High Throughput Computing capabilities and can efficiently exploit coarse-grain parallelism that is available in modular applications. With the emergence of many different general-purpose DCIs world-

Permission to make digital or hard copies of all or part of this work for personal or classroom use is granted without fee provided that copies are not made or distributed for profit or commercial advantage and that copies bear this notice and the full citation on the first page. To copy otherwise, to republish, to post on servers or to redistribute to lists, requires prior specific permission and/or a fee.

WORKS'10, November 14, 2010, New Orleans, USA.

Copyright 2010 ACM X-XXXXX-XX-X/XX/XX ...\$10.00. wide, among which the European $\mathrm{EGI}^{1}$ and the US $\mathrm{OSG}^{2}$, scientists are interested in porting their applications to different resources to improve performance. As an abstraction for describing distributed applications, workflows ease the porting of applications on different DCIs. However, application performance is highly dependent on the DCI capacities, the middleware installed, its access policies and the resources available. Furthermore, different DCIs may exhibit very different operation models whose impact on applications performance is difficult to anticipate. As a consequence, making an objective comparison between different DCIs is a difficult problem that requires significant investment and experimental methodology.

Dedicated tools have been developed to model and make predictions on distributed tasks execution time (e.g. Performance Analysis and Characterisation Environment - PACE $[9,10])$ and network traffic (e.g. Network Weather Service - NWS $[13,14])$ that are used for decision making within middleware. However these approaches are difficult to exploit in the case of large scale distributed infrastructures due to the complex interaction of multiple computing and networking resources and the external, uncontrollable workload resulting of other system users activity. Some probabilistic models attempt to model the overall DCI operational behavior [5, 3, 6]. These approaches have not been extended to tackle performance assessment issues though, especially in the context of multiple DCIs joint modeling.

The method adopted in this paper is based on experimental measurements instead, through execution of a same workflow-based application on the two DCIs to compare. It exploits the GWENDIA data-intensive grid workflow language designed to cover a broad class of distributed scientific applications [7], as well as two enactors supporting this language but implementing interfaces to different execution DCIs. MOTEUR ${ }^{3}$ is a GWENDiA language interpreter interfaced to the EGI infrastructure's gLite middleware, while DIET MA DAG ${ }^{4}$ is transforming GWENDIA workflows into Directed Acyclic Graphs and using specialized schedulers from the DIET middleware to submit computation tasks to

\footnotetext{
${ }^{1}$ European Grid Initiative, http://www.egi.eu

${ }^{2}$ Open Science Grid, http://www.opensciencegrid.org

${ }^{3}$ http://modalis.polytech. unice.fr/softwares/moteur

${ }^{4}$ http://graal.ens-lyon.fr/DIET/
} 
the Grid'5000 French research infrastructure. To acquire objective information on the DCIs, various time measurements are performed during workflow execution, that cover the CPUs performance, data transfer, and middleware overhead:

- Application makespan: the execution completion time, as experienced by the user.

- Processes execution time: the average execution time of all tasks executed within the workflow.

- Data transfer time: the average input/output data transfer time of all tasks executed within the workflow.

- Processes idle time: the average idle time (including all delays such as scheduling decision time, queuing time, etc) experienced by all tasks executed within the workflow.

This case study is based on EGI (formerly known as EGEE) and Grid'5000, two DCIs that have been developed to fulfill different purposes and operating completely different middleware. Grid' 5000 intends to be an instrument for research in computer-science (a "grid telescope") while EGI is meant to be a production platform for e-Science. These two DCIs exhibit different capacities and deliver variable quality of service. Furthermore, DCIs are by nature shared infrastructures which undergo variable and uncontrollable workload conditions depending on the concurrent usage of the same infrastructure by different users. An infrastructure should therefore be evaluated on the functionality it provides to its users as well as performance. No acknowledged model, benchmark or simulator exists for production grids although some efforts attempted to describe part of them $[1,4]$. It is therefore necessary that the performance comparison study is based on an extensive set of experiments. Setting up comparable experimental conditions between the two target platforms is a non-trivial issue. Still, performance measurements should be interpreted with flexibility, given that they are subject to unpredictable concurrent activities causing variations. In particular, averaging the results produced by multiple experiment runs lowers the variations observed.

In the reminder, the two target infrastructures are detailed and compared qualitatively in Section 2. The workflowbased experimental framework for achieving objective performance comparison on large-scale DCIs is then introduced in Section 3 and comparison results between EGI and Grid'5000 are reported in Section 4. The impact of the methodological framework used for the experiments is discussed in Section 5.

\section{OVERVIEW OF THE TWO CONSIDERED DCIS}

Both the EGI and the Grid'5000 grid infrastructures are large-scale DCIs consisting of a federation of computing centers interconnected through a high-bandwidth network. However, there are very little common features between these infrastructures as detailed below. Their operation models and objectives differ significantly.

\subsection{EGI production grid}

EGI (formerly known as EGEE) is a pan-European productionquality grid infrastructure deployed for addressing the needs of multiple scientific communities. Operated as a production service for e-Science, there is a 24/7 maintenance of the infrastructure. The infrastructure experiences a permanent and variable workload resulting from the competitive usage of the resources available by a very large user community (more than 10,000 users are registered). The middleware installed is a stable release (gLite middleware) deployed on quite homogeneous operating systems (mostly Scientific Linux and CentOS 4 or 5 , i.e. free versions of the RedHat Enterprise Linux). It has a very large scale: currently 250 computing centers delivering more than 160,000 $\mathrm{CPU}$ cores and tens of $\mathrm{PB}$ of storage space mutualize their resources. The clusters are managed by heterogeneous batch schedulers (PBS, LSF, etc) and the gLite middleware provides some glueing capability including a shared security infrastructure, a Workload Management System on top of the local resource managers, and a file catalog to produce a virtual grid-wide file hierarchy.

\subsection{Grid'5000 research grid}

Grid'5000 is an experimental grid infrastructure dedicated to research in computer science on large-scale distributed systems. It is composed of 9 sites interconnecting heterogeneous clusters through a dedicated high-performance network. More than 5000 CPU cores are available. Grid'5000 resources are completely reconfigurable for the need of experimentations. It is possible to reserve a fraction of the nodes available and to deploy any operating system and tool on these resources. As such, Grid'5000 does not operate a specific middleware stack. A rich tooling is available to reserve resources and deploy custom system images though. In the experiments reported in this paper, Scientific-Linux-4 system images have been deployed on the reserved Grid'5000 resources and the DIET [2] middleware has been installed.

\subsection{Qualitative comparison}

Beyond raw performance, grid usability depends on the features available to support application executions. This section summarizes the main differences between EGI and Grid'5000 on this aspect.

\section{Production usage.}

The EGI infrastructure is dedicated to production. As such it exposes to the users a sustained service with guaranteed access to the resources over a very long period of time. In particular, storage resources are provided for longterm storage of scientific data and resources availability is guaranteed (up to the competitive use by different users). Conversely on the Grid'5000 infrastructure, resources are dedicated for the time of an experiment. Resources are then freed, and scratch data storage is not guaranteed for longer time period (permanent data has to be saved to the persistent shared space of the platform). Another major difference between production and research infrastructures is the workload: it is completely dependent on the usage of other users on a production infrastructures while it is much more stable on dedicated resources. There might be side effects coming from the activity of other users on dedicated resources though. In particular, the network traffic is impacted by communications independent from the experiment to which the resources are dedicated and the allocation of multiple CPUs or multiple cores from the same board to different experiments may lead to undesirable run conditions (mem- 


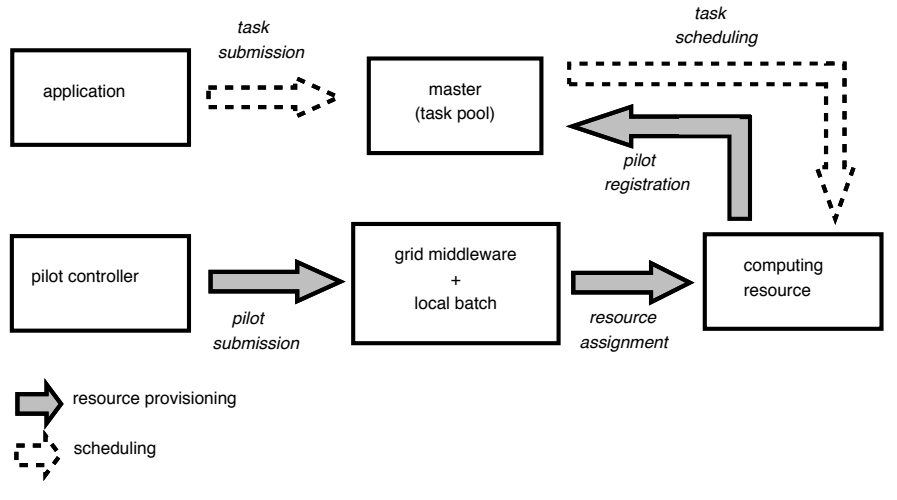

Figure 1: Principle of a pilot-job execution.

ory shortage due to concurrent use of the central memory, performance side effects due to I/O bottlenecks...).

\section{Resource reservation.}

The EGI infrastructure operates a collection of batch systems coordinated through a Workload Management System. The latter implements a meta-scheduler over heterogeneous batch schedulers. Conversely, the Grid'5000 infrastructure relies on resource reservation: resources are reserved in advance for a pre-determined duration. The allocated resources are fully dedicated to the user who made the reservation. Consequently, each task submitted to the EGI infrastructure undergoes a variable delay related to the time needed for the match-maker to identify a target batch system, the batch system queuing time and data transfer delays. On the Grid'5000 infrastructure, tasks submitted are potentially executed immediately (if sufficient resources were reserved) and undergo only scheduling and data transfer time. Furthermore, the amount of resources available for an application is well known and controlled, while on the EGI infrastructure it is difficult to measure the number of resources available for a specific application. Only the possible number of accessible resources is known, but in practice these resources are shared among the users. To improve resource dedication on the EGI infrastructure, pilot job techniques have been widely used $[8,11,12]$. As shown on Figure 1, pilot jobs are submitted through the regular job submission system. However, pilots are not regular application jobs. Once started, they keep occupying the resource, connecting to an application-level master node to request for application tasks to be executed. If enough tasks are available, they keep on processing tasks as much as the resource can be kept (i.e. as long as the pilot does not get killed by the batch system). This strategy proved very efficient from a user perspective as the pilots allocated are fully dedicated to the submitter. The pilot submission (resource provisioning) is still dependent from the batch submission system though.

\section{Scale.}

The EGI infrastructure gathers more than $140,000 \mathrm{CPU}$ cores distributed over 250 sites world-wide while the Grid' 5000 infrastructure has some 5,000 CPU cores distributed over 9 sites in France. The scale and complexity of these infrastructures significantly differ, although both rely on WAN interconnection. From a user point of view, the scale factor can- not be interpreted as a raw performance indicator since the EGI resources undergo competition from much more users.

\section{Middleware.}

While the EGI middleware is imposed by the infrastructure, the Grid'5000 infrastructure appears much more flexible and customizable for application needs. In addition, users can acquire root access to allocate nodes from Grid' 5000 while it is not the case on EGI nodes. The EGI Data Management Service provides user with a grid-wide virtual file hierarchy while data in Grid'5000 is clustered per site, each site operating an NFS server.

\section{Heterogeneity.}

Both infrastructures have different (and evolving) CPU types deployed. The EGI infrastructure is more homogeneous as it only features Intel-compatible hosts operating RedHat-based operating systems. The main differences between clusters are due to different generations of CPUs (32 versus 64 bits, mono versus multi-cores, etc). The Grid' 5000 infrastructure has a wider variety of CPUs and architectures installed.

\section{Security.}

A consequence of the flexibility provided by the Grid'5000 administration capability is the stricter security policy. On Grid'5000 the network is completely closed: all outgoing connections are filtered and inbound connectivity is limited to ssh access to cluster gateways (one per site). Conversely, the network is open within the Grid'5000 infrastructure, even between hosts located in different sites. The situation is different on EGI. Generally, outbound connectivity is allowed by sites although nothing is formally specified. Yet, most of sites deny incoming connections from external networks, even from other EGI sites. These connectivity differences have serious implications on application deployment. The security infrastructure on EGI is based on a shared Public Key Infrastructure and the delivery of X.509 certificate by a network of Certification Authorities trusted grid-wide. Regular (yet synchronized) Unix accounts are created for each Grid'5000 site-wise. As a result, the authorization framework differs on both infrastructures. For instance, regular Unix file permissions are used on Grid' 5000 while a specific ACL-based file access control is implemented in the EGI Data Management System. The need for data and user security are lower in Grid'5000 since the resources are only allocated for short period to each experiment and the network is completely closed to the outside world.

\section{Reliability.}

Grid infrastructures and middleware stacks encounter reliability problems proportionally to their complexity and scale. Users of the EGI infrastructure usually report high error rates (up to $30 \%$ in some cases, regularly more than $10 \%)$. These result in non negligible amounts of failed tasks and in high and unforeseeable delays in the tasks management time (the tasks undergo delays that are typically distributed as heavy-tailed probability laws).

\section{EXPERIMENTAL SETUP}

To quantitatively compare executions on the EGI and the Grid'5000 infrastructures, different application performance 
metrics are measured along several runs of the same application. The experiments described below attempt to minimize the differences between the infrastructure when it can be controlled.

A simple application workflow, illustrated in Figure 2, was selected for all runs. The workflow performs segmentation of the myocardium (inner and outer walls of the heart left and right ventricles) in $3 \mathrm{D}+\mathrm{T}$ cardiac image sequences using a physical elastic template of the myocardium. The goal of this workflow is to optimize the quality of the segmentation result based on an exhaustive sweep of the parameter space. In Figure 2 the two first processing steps (mhd2qc and ImgAndModelInit) are executed only once at the beginning of each run to initialize the segmentation process. The subsequent segmentation step (det3D4) is executed once for each set of segmentation parameters specified as input. There is a combinatorial number of runs depending on the number of parameter values (in particular, different model attraction force factors (force workflow input), and different values of the elastic template physical Poisson and Young coefficients, P0, P1, Y0, and Y1). This workflow can produce a variable number of computing tasks depending on the size of the considered parameter space. Three experiment sizes were considered in these experiments: a small-size experiment, producing 14 tasks; a medium-size experiment, producing 202 tasks; and a large-size experiment with 2082 tasks.

To obtain comparable execution environments, Scientific Linux (v4) operating system images have been deployed on the Grid'5000 infrastructure. As a consequence, the same application binaries can be invoked on both platforms. Each experiment is restarted 5 times, at various times and days, to average measurements and estimate standard deviations.

\subsection{Relative performance comparison}

The first experiment provides information of relative grid performance by executing the same application in controlled conditions to align the execution environments as much as possible while running on different grid infrastructures. Pilot jobs are used on the EGI infrastructure, making it possible to dedicate a controllable number of resources for the application run. The pilots are started before the execution runs and an external process monitors the population of pilot jobs to maintain its number.

In these experiments the target is to maintain a total number of 54 pilots. To achieve this, a dedicated "buffer" scheduler was developed in the DIANE pilot-job framework [8]. It behaves as a connection buffer between the pilots and the "real" scheduler used for the experiment. An overestimated number of pilots are submitted before the experiment starts and the buffer scheduler progressively delivers pilot connections to the real scheduler to ensure that exactly 54 resources are available for the experiment. In practice, the provisioning of up to 90 pilots was required to ensure a constant pilot population size during the longest experiments. The pilots are located on pre-determined sites to ensure that resources distribution keeps coherent along the experiment. Similarly, data transfer conditions were adjusted. Coherently with the policy set in the DIET DAGDA (file persistence), files transferred to a pilot node are cached so that a subsequent task using the same file does not require an additional grid file transfer. Output files are stored on the local site's Storage Elements to minimize transfer times.

The same number of resources is allocated on EGI and

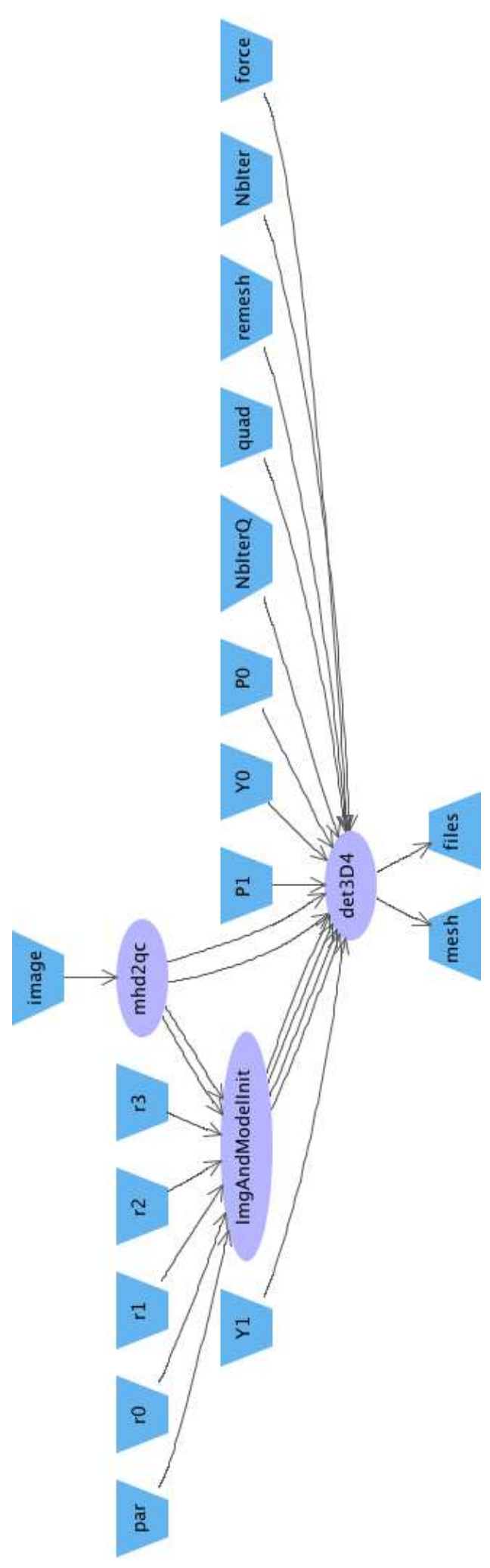

Figure 2: Segmentation workflow graphical representation 


\begin{tabular}{|c|c|c|c|}
\hline & & Grid'5000 & EGI \\
\hline \multirow{3}{*}{ Workload } & Resource reservation & done by OAR & mimicked by pilot buffering \\
\hline & Task transfer & direct connection to SeDs & active polling from pilots to master \\
\hline & Error management & errors do not occur & $\begin{array}{c}\text { faulty pilots are removed, buffer has failover, } \\
\text { MOTEUR resubmit tasks }\end{array}$ \\
\hline management & Nodes location & some workers are on the master's site & workers and master are on different sites \\
\hline \multirow{3}{*}{$\begin{array}{c}\text { Data } \\
\text { management }\end{array}$} & Input files location & pre-installed & replicated on several sites \\
\hline & Input file transfers & cached by DAGDA on the local site & cached by each pilot on the worker node \\
\hline & Result files & stay on the host that produced them & transferred to the site's SE \\
\hline
\end{tabular}

Table 1: Conditions of the experiment

Grid'5000 (54 CPU cores). Two experimental setups were considered: in the first one, 54 dedicated resources were co-allocated on a single site while in the second setup 3 different sites (each hosting 18 resources) were used to study the impact of wide area network communications. The application makespan, the processes execution time, the data transfer time and the processes idle time are measured. The execution being performed in similar conditions, with a controlled number of resources all dedicated to the experiment, the time measures can be compared and give some insight on the performance difference resulting from the execution environment (workflow managers, middleware, and schedulers).

\subsection{Production conditions}

A user exploiting the infrastructures with no particular setup controlling the number of resources will experience performance differences resulting from the uncontrolled number of computing resources available (in addition to other structural differences). This experiment compares the performance achieved when running large-size applications on the EGI infrastructure in controlled conditions (using pilots and controlled reservations) to the performance obtained in regular production (still using pilots but greedy pilot submission).

The larger the number of heterogeneous resources used within an experiment, the higher the probability for failure. This experiment also quantifies the loss induced by the use of a large-scale production environment versus execution on a limited number of well controlled resources.

\section{QUANTITATIVE COMPARISON}

Figure 3 displays the job graph of a typical run on the EGI (top) and the Grid'5000 (bottom) DCIs for a smallsize experiment, in controlled conditions as described in Section 3.1. Horizontally, tasks appear in the order they are created. The first task created is numbered 0 . It corresponds to the mhd2qc process. After approximately 200 seconds, the process terminates and the subsequent task ImgAndModelInit (task number 1) starts. When this task terminates, the 12 segmentation tasks start concurrently (tasks 2 to 13). Since there are more computing nodes than needed, all tasks are supposed to start immediately. Their execution time is similar and all complete approximately at the same time. For each of the 14 tasks, the job graph shows the time spent before completion, split in five successive time slots:

- white: time before the task is ready (i.e. all its predecessors completed);
- brown: idle time of the task (including scheduling time and queuing time, once the task has been created);

- blue: input data download time;

- green: running time of the task; and

- purple: output data upload time. The actual values are reported in Table 2.

The variations on the execution time of the segmentation tasks are partly explained by the non-deterministic nature of the segmentation algorithms (two runs with different input parameter values may result in slightly different execution times), and partly by the heterogeneity of the CPU cores executing the codes. On EGI, the 12 segmentation tasks were executed on Intel Xeon CPUs with slightly different frequencies $(2.27,2.50$ and $2.83 \mathrm{GHz}$ ), while on Grid'5000 they were executed on more heterogeneous resources ranging from Intel Xeon L5420 @ 2.5GHz (highest) to AMD Opteron $245 @ 2 \mathrm{GHz}$ (lowest). Execution time is significantly lower on the EGI nodes than on Grid' 5000 ones as hardware resources operated in production computing centers tend to be renewed more frequently, leading to higher performing computing nodes.

Conversely, despite the controlled conditions enforced on the EGI grid infrastructure, the middleware overhead (idle time) and the data transfer times are orders of magnitude higher on EGI than on Grid'5000. The gLite middleware secured communication protocols and polling loops introduce non-negligible latencies in the management of the jobs and the access to files. Moreover, the gLite data management system is a multi-stacked system (mainly composed of LFC, SRM and gridFTP) which introduces significant latencies on the data transfers. On Grid'5000, idle times and data transfer times are negligible. The tasks push strategy implemented by the DIET middleware does not cause artificial delays as observed when using pilots on EGI. The data transfers on the infrastructure dedicated high performance network and the file caching strategy implemented in DIET are efficient to lower data transfer times.

\subsection{Medium-size experiments}

Figure 4 shows the job graph of a typical medium-size run on both DCIs and Table 3 gives quantitative values averaged over several runs. The clustering of tasks in batches of 54 can clearly be seen on EGI: only 54 nodes are available at any time and 54 tasks are executed concurrently, appearing as 4 execution phases in the job graph (with a progressive de-sequencing due to the heterogeneous data transfer and execution times). On Grid'5000, the 4 batches of 54 concurrent tasks appear vertically (4 groups of execution). The 


\begin{tabular}{|l|c|c|c|c|}
\hline DCI & Makespan & Idle time (brown) & Data transfer time (blue and purple) & Execution time (green) \\
\hline EGI & $965 \mathrm{~s}$ & $33 \mathrm{~s} \pm 18$ & $132 \mathrm{~s} \pm 29$ & $275 \mathrm{~s} \pm 93$ \\
\hline Grid'5000 & $1061 \mathrm{~s}$ & $0.21 \mathrm{~s} \pm 0.34$ & $3.37 \mathrm{~s} \pm 2.57$ & $541 \mathrm{~s} \pm 298$ \\
\hline
\end{tabular}

Table 2: Execution behavior of a small-size experiment on EGI and Grid'5000.
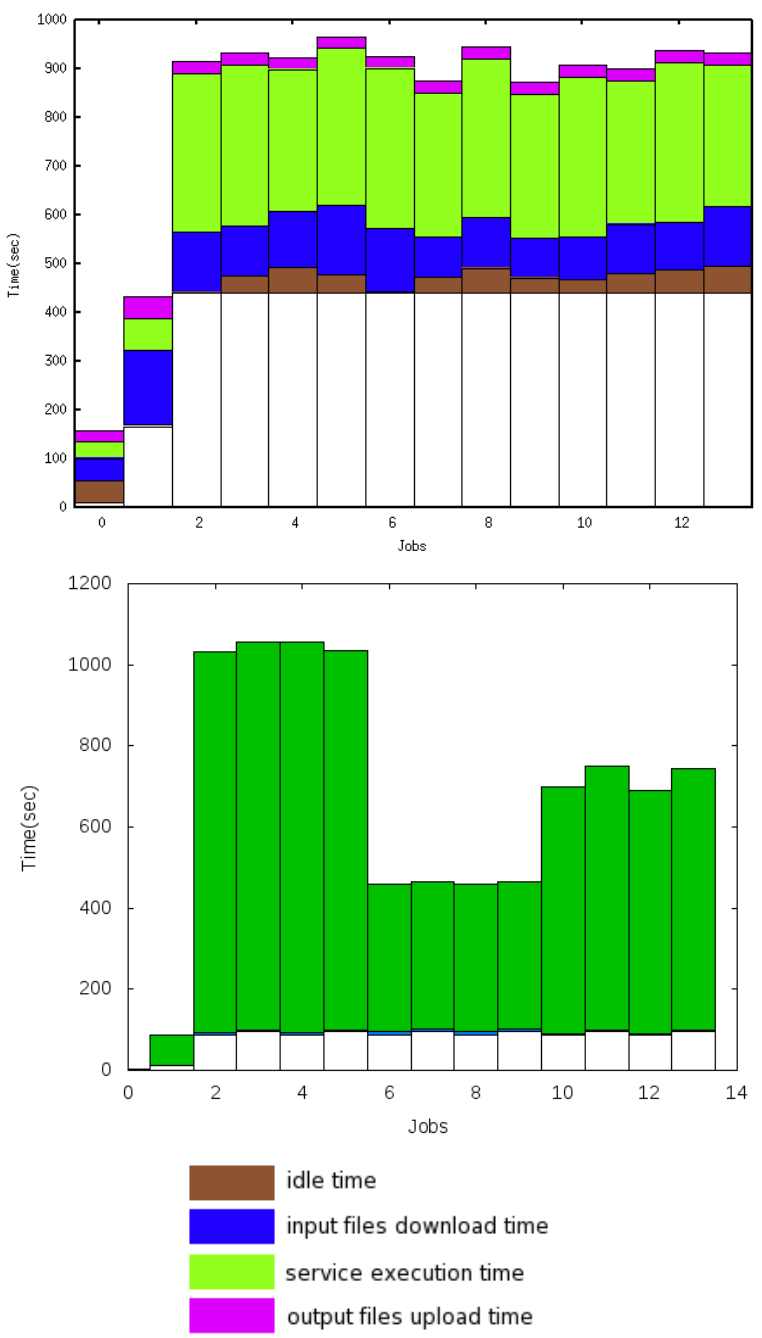

Figure 3: Typical job graph for a run with 14 tasks on the EGI (top) and Grid'5000 (bottom) DCIs. horizontal slicing of the job graph is due to the DIET MADAG strategy to handle large workflows as the complete 202-task workflow is sliced into 10 sub-DAGs which compete to access resources available. The figure also clearly shows the impact of the EGI pilots file caching strategy: the first batch of 54 tasks transfer input files (blue time slot) which are then cached on the computing nodes. Since other segmentation tasks will reuse the same files and run on the same allocated nodes, there is no more input file transfer needed from the $55^{\text {th }}$ task.

On the EGI the profiles of mono-site and 3-site runs are close to each other. For 3 sites, a few task failures caused task resubmissions. In spite of controlled conditions, there are significant variations on the production infrastructure mostly due to data transfer time heterogeneity. The impact of using 3 sites instead of 1 is far greater on Grid'5000 than it is on EGI. On Grid'5000, the input data transfer times impact becomes non-negligible in the case of multi-site runs. On EGI, the lack of difference in the data transfer performance is explained by the fact that input data files were replicated on several sites of the infrastructure. Data transfers over WANs may occur in any case, e.g., if the chosen site does not host the input data. The similar values observed in the idle time come from the fact that in any case the master and the pilots have to be deployed on different network domains, which imposes an active polling from the pilots to the master to circumvent connectivity policies. Execution times are also very much similar, due to the relative homogeneity in the CPU characteristics of the different sites. This tends to suggest that increasing the number of EGI sites does not significantly burden the performance. Surprisingly, the variability of the makespan is higher on Grid'5000 than it is on EGI. It suggests that the reproducibility of experiments on Grid'5000 is not better than using the controlled setup on EGI. The standard-deviation of the idle time and data transfer time is of the same order of magnitude as the average while it is at least twice lower on EGI.

\subsection{Large-size experiments}

Figure 5 and Table 4 report results on large-size experiments. The density of tasks makes the overall job graph hardly readable: only the linear profile of job completion can be perceived. This behavior is expected as a constant number of computing nodes is available. On Grid'5000, the profile is a bit sharper due to heterogeneity on computing resources performances. The reproducibility of mono-site experiments is much better on Grid'5000 $(\sigma=13.4 s)$ than on EGI $(\sigma=1172 s)$. For 3-site experiments, the reproducibility is better on EGI though $(\sigma=287 \mathrm{~s}$ versus $641 \mathrm{~s}$ on Grid'5000).

It proved to be difficult to complete large-size runs (2082 tasks) in controlled condition on the EGI infrastructure. Many experiments failed before completion due to various problems (permanent file transfer errors, pilots being removed from sites for various reasons, grid unavailability prob- 


\begin{tabular}{|l|c|c|c|c|}
\hline DCI & Makespan & Idle time & Data transfer time & Execution time \\
\hline EGI, mono-site & $2299 \mathrm{~s} \pm 460$ & $627 \mathrm{~s} \pm 143$ & $81 \mathrm{~s} \pm 58$ & $317 \mathrm{~s} \pm 6$ \\
\hline EGI, 3-sites & $2226 \mathrm{~s} \pm 331$ & $576 \mathrm{~s} \pm 55$ & $58 \mathrm{~s} \pm 27$ & $335 \mathrm{~s} \pm 0.3$ \\
\hline Grid'5000, mono-site & $2028 \mathrm{~s} \pm 563$ & $27 \mathrm{~s} \pm 91$ & $0.97 \mathrm{~s} \pm 1.36$ & $430 \mathrm{~s} \pm 40$ \\
\hline Grid'5000, 3 sites & $3443 \mathrm{~s} \pm 388$ & $111 \mathrm{~s} \pm 223$ & $20 \mathrm{~s} \pm 15$ & $576 \mathrm{~s} \pm 240$ \\
\hline
\end{tabular}

Table 3: Execution behavior of medium-size experiments on EGI and Grid'5000. For each metric, the values measured are averaged over 3 to 5 workflow executions.
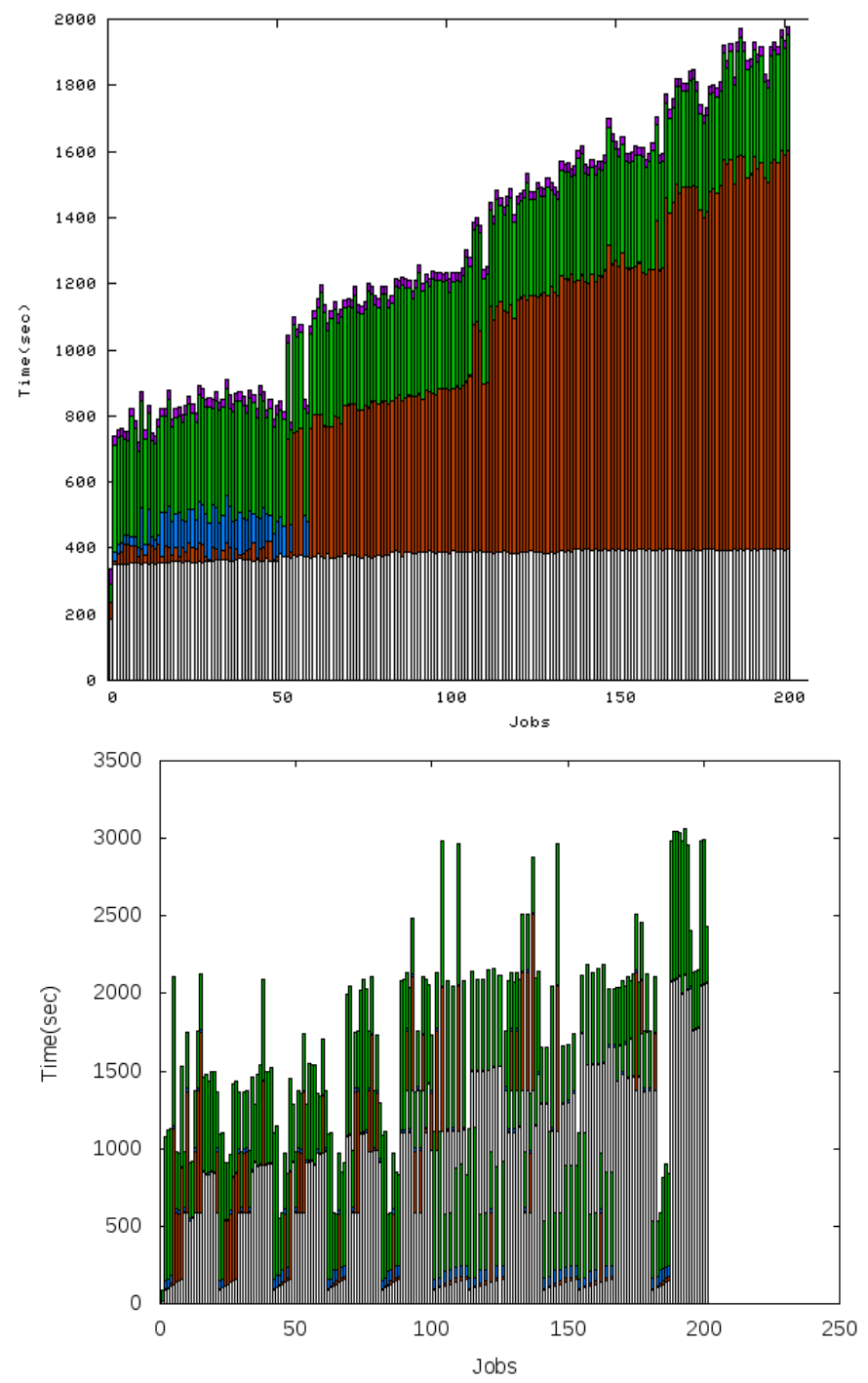

Figure 4: Typical job graph for a run with 202 tasks on the EGI (top) and the Grid'5000 (bottom) DCIs.
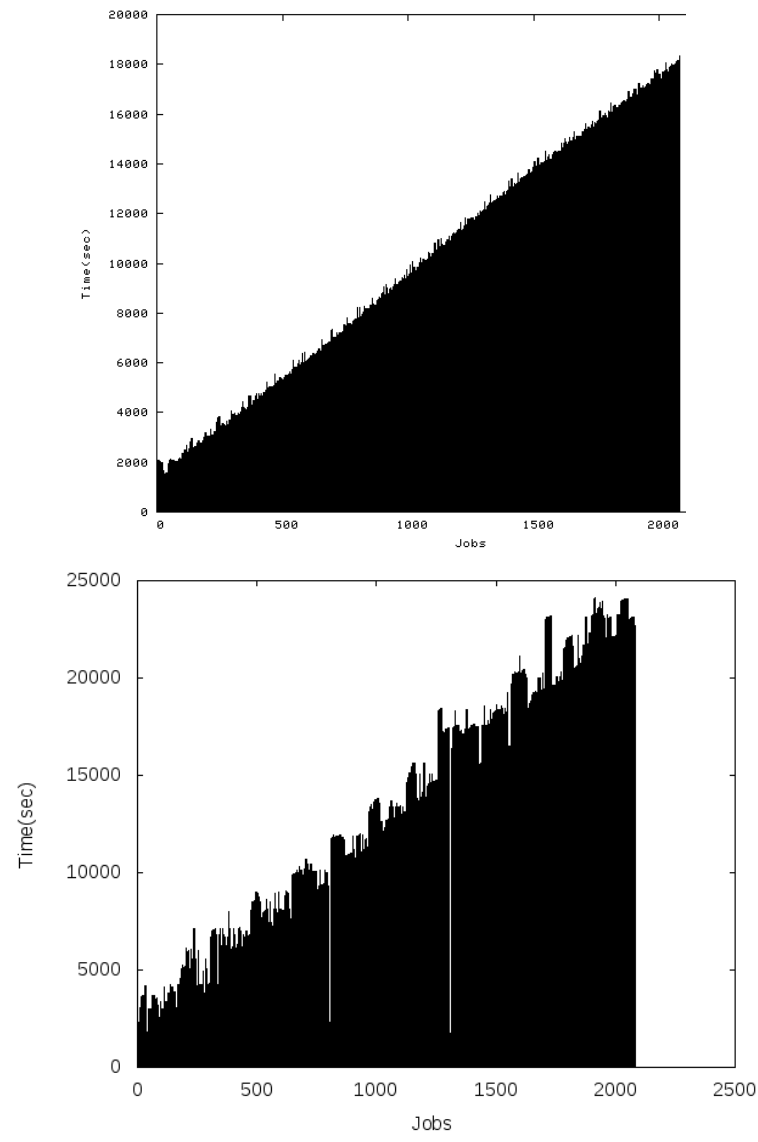

Figure 5: Typical job graph for a run with 2082 tasks on the EGI (top) and the Grid'5000 (bottom) DCIs.

lems, etc). The over-buffering strategy adopted to ensure a constant-size population of pilots along the complete experiment caused some resources to be reserved by idle pilots for the experiment duration ( 5 hours or more). Such pilots may be considered as faulty processes and be automatically removed to release nodes by the site administration procedures. Other causes of pilot failure increase with the duration of the experiment. Consequently, experiment failures are common. On Grid'5000 as well, some faults of the reservation system (reservation overlaps between multiple users) and a complete platform crash were experienced.

\section{CONTROLLED VERSUS UNCONTROL- LED EXECUTION CONDITIONS}

As described in Section 3.2, the performance in a con- 


\begin{tabular}{|l|c|c|c|c|}
\hline Site & Makespan & Idle time & Data transfer time & Execution time \\
\hline EGI, mono-site & $17214 \mathrm{~s} \pm 1172$ & $6851 \mathrm{~s} \pm 1099$ & $50 \mathrm{~s} \pm 24$ & $356 \mathrm{~s} \pm 1$ \\
\hline EGI, 3 sites & $19344 \mathrm{~s} \pm 287$ & $9577 \mathrm{~s} \pm 1003$ & $38 \mathrm{~s} \pm 10$ & $420 \mathrm{~s} \pm 18$ \\
\hline Grid'5000, mono-site & $24940 \mathrm{~s} \pm 13$ & $12 \mathrm{~s} \pm 86$ & $0.51 \mathrm{~s} \pm 1.24$ & $629 \mathrm{~s} \pm 25$ \\
\hline Grid'5000, 3 sites & $24477 \mathrm{~s} \pm 641$ & $70 \mathrm{~s} \pm 220$ & $13 \mathrm{~s} \pm 12$ & $594 \mathrm{~s} \pm 200$ \\
\hline
\end{tabular}

Table 4: Execution behavior of large-size experiments on EGI and Grid'5000. For each metric, the values measured are averaged over 3 to 5 workflow executions.

trolled environment is expected to vary when compared to regular production conditions, due to two antagonist factors: the limited number of controlled resources causing performance loss and a better control over the availability and homogeneity of resources causing performance improvement. These two factors are quantitatively evaluated in the experiments based on large-size runs reported in this section.

\subsection{Impact of pilots and limited number of re- served resources}

Although the experiments implemented above require to wait for a given number of pilots to become simultaneously available before starting execution, this delay is not implemented in production to avoid wasting CPU cycles. Figure 6 shows an experiment executed on the EGI infrastructure using Algorithm 1 to submit pilots. This is a greedy algorithm submitting pilots as long as tasks are scheduled in the master. The dispatching of pilots among grid sites is up to the gLite Workload Management System and not controlled here.

The profile of this curve significantly differs from the one observed in Figure 5. It is not linear any more: the workflow system is not limited to a restricted number of pilots and attempts to allocate concurrently as many resources as possible. It can be seen in Figure 6 that more resources are recruited than with 54 dedicated pilots, although these resources were not pre-reserved before the beginning of the experiment. As a result, it takes some time for the first tasks to be processed (slow start, with $\approx 2000$ s latency before the first task completes) but the number of resources recruited quickly compensate for the performance loss (high throughput with a low slope envelope curve) until the last and slowest tasks are processed (peak in the right-most part of the graphs). The peak comes from task resubmissions resulting from failures. When tasks fail towards the end of the experiment, only a few pilots remain available, which delays a lot the completion of the last task. This is even worse when tasks have to be resubmitted several times. This

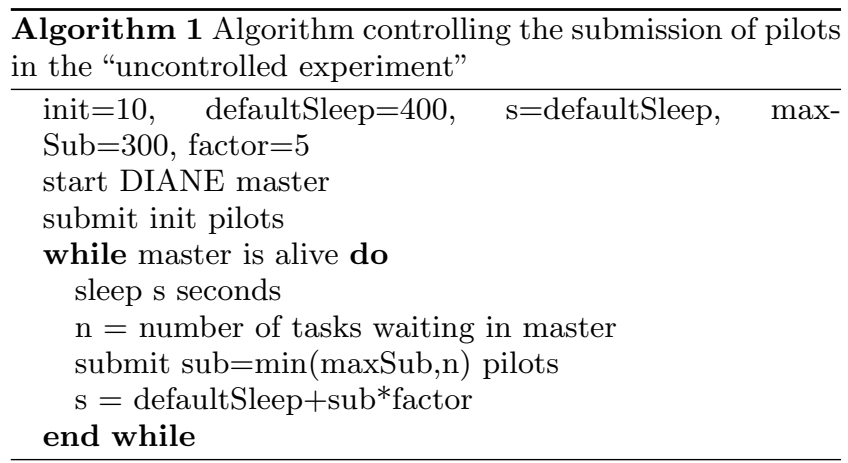

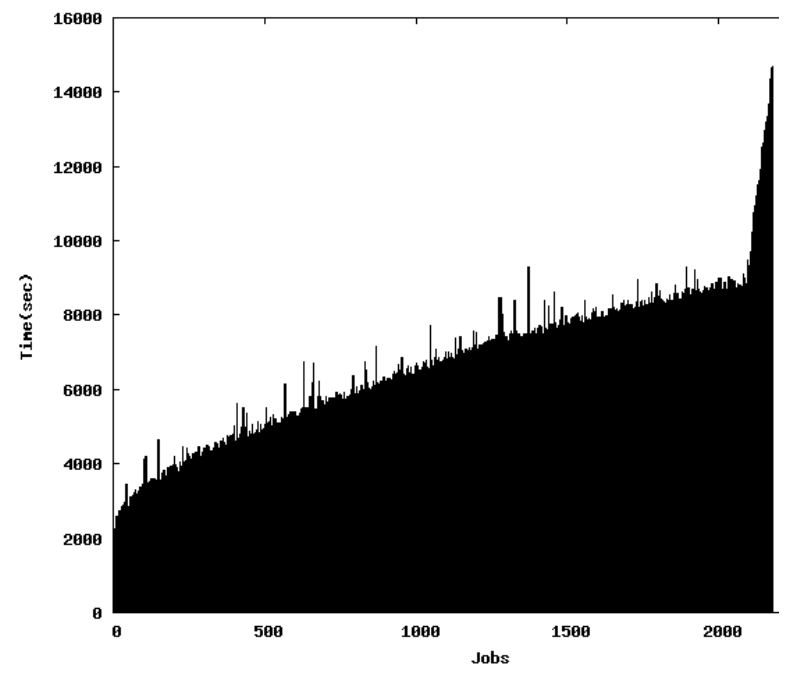

Figure 6: A run with 2082 tasks under regular production conditions.

phenomenon suggests that the sites involved in an experiment should be carefully controlled and probably limited to a smaller number of reliable, efficient sites.

Table 5 summarizes the behavior of this experiment. It can be noted that the average execution time is higher and has much higher variations than the runs in controlled condition using 1 or 3 sites (Section 4.2). This can be expected as the jobs are spawned over a large number of sites with different hardware resources. The average makespan of these experiments is slightly better, but in the same order of magnitude, than the makespan observed in controlled condition $(\approx 16000 \mathrm{~s})$. This shows that the set of resources available for execution (the biomed Virtual Organization resources) on the EGI grid infrastructure only slightly outperforms a set of 54 dedicated resources. This comment is only valid considering that:

- Unlike the set of dedicated resources, EGI resources are not dedicated to a single experiment at a time and multiple experiments can be ran concurrently with only very small performance impact (in other terms, the throughput can increase significantly although the absolute performance cannot);

- The EGI resources are shared between multiple users and disciplines;

- The EGI resources are administrated and available $24 / 7 / 365$ to the users, which is hardly the case of dedicated clusters installed in small laboratories. 


\begin{tabular}{|l|c|c|c|c|}
\hline Experiment & Makespan & Idle time & Data transfer time & Execution time \\
\hline $1^{\text {st }}$ run & 14690 & $5970 \mathrm{~s} \pm 1751$ & $64 \mathrm{~s} \pm 1277$ & $416 \mathrm{~s} \pm 166$ \\
\hline $2^{\text {nd }}$ run & 13655 & $4946 \mathrm{~s} \pm 1571$ & $78 \mathrm{~s} \pm 727$ & $416 \mathrm{~s} \pm 170$ \\
\hline $3^{\text {rd }}$ run & 16177 & $6092 \mathrm{~s} \pm 2102$ & $70 \mathrm{~s} \pm 773$ & $410 \mathrm{~s} \pm 118$ \\
\hline average & $14841 \mathrm{~s} \pm 1035$ & $5669 \mathrm{~s} \pm 514$ & $71 \mathrm{~s} \pm 5.5$ & $414 \mathrm{~s} \pm 3$ \\
\hline
\end{tabular}

Table 5: Execution behavior of large-size experiments on EGI, in uncontrolled conditions (using algorithm 1 to submit the pilots).

The reasons why the whole EGI infrastructure does not outperform significantly the set of dedicated resources lies in the variation of unit processes execution times and the failure rates encountered which is much higher than when using controlled pilots. Table 6 summarizes the distribution of tasks (number of site batch queues used during the experiment) and the failure rate observed in non-controlled conditions (number of failed jobs versus 2082 jobs to complete).

Finally, it should be noted that the workflow considered in these experiments is not very sensitive to failures: failures cause resubmission which lengthen the overall workflow termination but there is no cascade effect due to dependencies on the tasks executed. A more complex workflow would probably be more penalizing regarding execution in noncontrolled conditions.

These results also show the impact of the last tasks on the makespan of the executions performed in production conditions. Improving the scheduling (both resource provisioning and task placement) would much likely have a significant impact on the makespan. Obviously, the number of sites used for a given experiment has to be better controlled in production.

\subsection{Impact of pilots start time synchroniza- tion}

Since pilot jobs are submitted using the regular batch interface of the gLite Workload Management System, their execution start is subject to the variable workload and execution conditions on the EGI production grid infrastructure at the time of the experiment. For obtaining controlled conditions on EGI (54 pilots ready for concurrent processing), it was necessary to submit an over-provisioned number of pilots (typically 70 to 90 ) and to wait for at least 54 of them to become available. This process could take from a dozen of minutes to hours (some experiments were aborted before starting because the necessary number of pilots could not be acquired in less than two hours). Under normal production use of pilots, this delay should be taken into account in the application makespan computation. Usually, production runs are started without trying to synchronize the pilots. Figure 7 displays a typical example of a medium-size production run with unsynchronized pilot starts. It can be noted that the time to execute the two first workflow initialization stages is slightly longer than the average time observed for comparable experiments (see Section 4.1), probably due to the time for the first pilot to become available before starting processing. Once the two first initialization tasks have been completed, approximately 20 segmentation tasks start concurrently. This corresponds to the number of pilots that could be started while the initialization tasks executed. The remaining pilots become available progressively as the run progresses until all 54 pilots are available. The resulting makespan (2904 s) is slightly longer than comparable experiments.

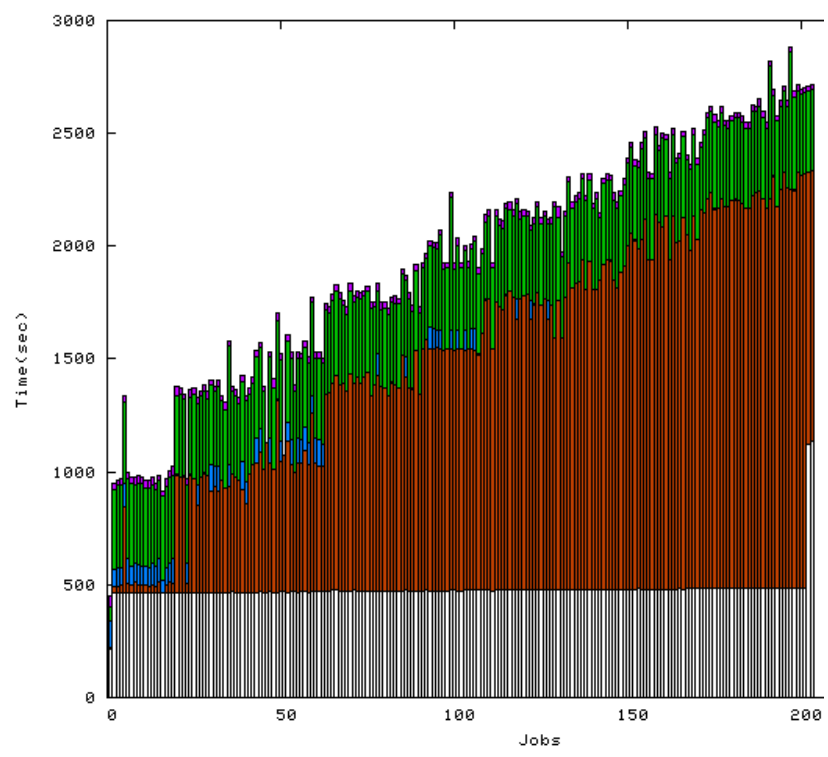

Figure 7: medium-size run without synchronization of the pilots start time.

\section{CONCLUSIONS}

This paper described an approach to compare the performance of different DCIs using language-compatible workflow engines to reproduce experiments on the targeted execution platforms. This method was applied to the EGI and the Grid'5000 DCIs which exhibit completely different objectives and architectures. To align execution conditions on both infrastructures, a co-reservation mechanism based on pilot jobs was implemented to allocate a fixed number of grid resources when no other mean of reservation is available. Results show that the approach can scale up to large-size experiments (thousands of jobs) although it seems difficult to continue far beyond due to practical problems arising (duration of experiments causing several technical problems and resources held idle for a long period of time).

Raw performance variations between the EGI and Grid'5000 infrastructures are clearly identified: the former benefits from latest computation hardware, demonstrating better performance than the latter (typically twice faster) while longest range communications and non-dedicated network cause data transfers to be less efficient. The experiments also exhibits differences between the gLite and the DIET middleware operating the resources on EGI and Grid'5000 respectively. 


\begin{tabular}{|l|c|c|c|c|}
\hline Experiment & \# batch queues & Total \# jobs & \# failed jobs & Failure rate \\
\hline $1^{\text {st }}$ run & 32 & 2180 & 98 & $4.71 \%$ \\
\hline $2^{\text {nd }}$ run & 33 & 2144 & 62 & $2.98 \%$ \\
\hline $3^{\text {rd }}$ run & 18 & 2108 & 26 & $1.25 \%$ \\
\hline
\end{tabular}

Table 6: Failure rates experienced in production in uncontrolled conditions.

gLite suffers much higher overhead related to security policies enforcement and polling strategies implemented.

The results reported here show that it is possible to conduct experiments related to task-based workflow execution on a production infrastructure with similar reproducibility than on an experimental platform. The controlled conditions maintained on the EGI infrastructure through pilots jobs are not completely realistic in production mode, due to the over-provision of pilots and the requirement for simultaneous co-allocation. However, many large-size experiments started using pilots on the EGI grid infrastructure to solve job latency and resource reliability problems. These applications are running in conditions close to the one enforced for these experiments and the variations are investigated in Section 5 .

In the future, it would be interesting to execute different workflows within the same framework to show the reproducibility or the variations of the measurements performed under such controlled conditions while dealing with different data flow patterns.

\section{ACKNOWLEDGMENTS}

This work is supported by the French ANR GwENDIA project $^{5}$ under contract number ANR-06-MDCA-009, and the European I3 SHIWA project ${ }^{6}$ under contract number 261585 .

\section{REFERENCES}

[1] W. H. Bell, D. G. Cameron, A. P. Millar, L. Capozza, K. Stockinger, and F. Zini. Optorsim: A Grid Simulator for Studying Dynamic Data Replication Strategies. International Journal of High Performance Computing Applications (IJHPCA), 17(4):403-416, Nov. 2003.

[2] E. Caron and F. Desprez. DIET: A Scalable Toolbox to Build Network Enabled Servers on the Grid. International Journal of High Performance Computing Applications, 20(3):335-352, 2006.

[3] T. Glatard, J. Montagnat, and X. Pennec. A probabilistic model to analyse workflow performance on production grids. In T. Priol, L. Lefevre, and R. Buyya, editors, IEEE International Symposium on Cluster Computing and the Grid (CCGrid'08), pages 510-517, Lyon, France, May 2008. IEEE.

[4] A. Legrand, M. Quinson, K. Fujiwara, and H. Casanova. The SimGrid Project - Simulation and Deployment of Distributed Applications. In Proceedings of the IEEE International Symposium on High Performance Distributed Computing (HPDC-15), Paris, France, 2006. IEEE Computer Society Press.

${ }^{5}$ GWENDIA, http://gwendia.polytech.unice.fr

${ }^{6}$ SHIWA, http://www. shiwa-project.eu
[5] D. Lingrand, T. Glatard, and J. Montagnat. Modeling the latency on production grids with respect to the execution context. Parallel Computing (PARCO), 35(10-11):493-511, Oct. 2009.

[6] E. Medernach. Workload Analysis of a Cluster in a Grid Environment. In Job Scheduling Strategies for Parallel Processing (JSSPP), pages 36-61, Cambridge, MA, USA, June 2005.

[7] J. Montagnat, B. Isnard, T. Glatard, K. Maheshwari, and M. Blay-Fornarino. A data-driven workflow language for grids based on array programming principles. In Workshop on Workflows in Support of Large-Scale Science (WORKS'09), pages 1-10, Portland, USA, Nov. 2009. ACM.

[8] J. T. Mościcki. Distributed analysis environment for HEP and interdisciplinary applications. Nuclear Instruments and Methods in Physics Research A, 502:426-429, 2003.

[9] G. Nudd, D. Kerbyson, E. Papaefstathiou, S. Perry, J. Harper, and D. Wilcox. PACE: A toolset for the performance prediction of parallel and distributed systems. International Journal of High Performance Computing Applications (IJHPCA), 3(14):228-251, Aug. 2000.

[10] D. Spooner, J. Cao, S. Jarvis, L. He, and G. Nudd. Performance-aware Workflow Management for Grid Computing. The Computer Journal, 3(48):347-357, May 2005.

[11] D. Thain, T. Tannenbaum, and M. Livny. Distributed computing in practice: the Condor experience. Concurrency and Computation: Practice 83 Experience (CCPE), 17(2-4):323-356, 2005.

[12] A. Tsaregorodtsev, M. Bargiotti, N. Brook, A. C. Ramo, G. Castellani, P. Charpentier, C. Cioffi, J. Closier, R. Diaz, G. Kuznetsov, Y. Li, R. Nandakumar, S. Paterson, R. Santinelli, A. Smith, M. Miguelez, and S. Jimenez. DIRAC: a community grid solution. Journal of Physics: Conference Series, 119, 2008.

[13] R. Wolski. Experiences with predicting resource performance on-line in computational grid settings. ACM SIGMETRICS Performance Evaluation Review, 4(30):41-49, Mar. 2003.

[14] R. Wolski, N. Spring, and J. Hayes. The network weather service: a distributed resource performance forecasting service for metacomputing. Future Generation Computer Systems (FGCS), 5-6(15):757-768, Oct. 1999. 